\title{
Effect of Noncollinear Magnetic Order on the Hyperfine Interactions in $\mathrm{Pt}_{3} \mathrm{Fe}$
}

\author{
A. Woźniakowski ${ }^{a}$, J. DeniszCZYK ${ }^{a, *}$ AND K. SzYMAŃsKi ${ }^{b}$ \\ ${ }^{a}$ Institute of Materials Science, University of Silesia, 40-007 Katowice, Bankowa 12, Poland \\ ${ }^{b}$ Faculty of Physics, University of Białystok, 14-424 Białystok, Poland
}

\begin{abstract}
The investigations of the hyperfine interactions in $\mathrm{Pt}_{3} \mathrm{Fe}$ with the noncollinear magnetic order by means of $a b$ initio methods are presented. It is shown that the ground state magnetic order of $\mathrm{Pt}_{3} \mathrm{Fe}$ is of noncollinear character. Electric field gradient and hyperfine magnetic field at ${ }^{57} \mathrm{Fe}$ nucleus were calculated and their dependence on the strength of Coulomb correlation interaction is discussed. The calculated values of electric field gradient correspond with the experimental data and the best agreement was found when the enhanced Coulomb correlation interaction for $3 d$-states of Fe was considered. Lattice, local valence electron $(3 d, 4 p)$ and weakly bound $3 p$ semicore electron contributions to electric field gradient are separated out and discussed.
\end{abstract}

PACS: 71, 71.20.Be, 71.20.Lp, 75.50.Bp, 76.80.+y

\section{Introduction}

Magnetic properties of Pt-Fe alloys depend strongly on concentration of the iron atoms. In disordered alloys the ferromagnetic long range order coexists with a weak antiferromagnetic component [1]. Among the Pt-Fe alloys, the magnetic structure of ordered $\mathrm{Pt}_{75} \mathrm{Fe}_{25}$ alloy (referred hereafter as $\mathrm{Pt}_{3} \mathrm{Fe}$ ) is most interesting and, despite intensive studies, is still not determined uniquely. $\mathrm{Pt}_{3} \mathrm{Fe}$ crystallizes in cubic $\mathrm{Cu}_{3} \mathrm{Au}\left(\mathrm{L1}_{2}\right)$ structure. Early neutron diffraction studies [2] have shown that below $T_{N}=160 \mathrm{~K}$ the $\mathrm{Pt}_{73,3} \mathrm{Fe}_{26,7}$ exhibits the long range antiferromagnetic order of $(1 / 2,1 / 2,0)$-type $\left(\mathrm{AFM}_{2}\right)$, but with decreasing temperature, at around $T_{S}=110 \mathrm{~K}$, the component of antiferromagnetic order of $(1 / 2,0,0)$-type $\left(\mathrm{AFM}_{1}\right)$ develops. Recent neutron scattering investigations of $\mathrm{Pt}_{3} \mathrm{Fe}$ have shown that below $T_{S}$ the noncollinear magnetic order develops in the compound [3].

In our previous paper [4] we presented the low temperature $(T=13 \mathrm{~K})$ Mössbauer effect and ground state $a b$ intio studies of $\mathrm{Pt}_{3} \mathrm{Fe}$ with the ferromagnetic, antiferromagnetic $\left(\mathrm{AFM}_{1}\right.$ and $\left.\mathrm{AFM}_{2}\right)$ magnetic order. The data discussed there have shown that the ground state magnetic structure of $\mathrm{Pt}_{3} \mathrm{Fe}$ is neither of $\mathrm{AFM}_{1}$ nor $\mathrm{AFM}_{2}$ type. The results of the ground state calculations of hyperfine interactions for $\mathrm{AFM}_{2}$ type of magnetic order agree qualitatively well with the low temperature experimental data, what, on other hand, was at variance with the neutron diffraction results, indicating the $\mathrm{AFM}_{1}$-type of magnetic order in $\mathrm{Pt}_{3} \mathrm{Fe}$ at low temperatures.

In order to clarify the discrepancy we included the noncollinear type of magnetic order into our ab initio investigations of $\mathrm{Pt}_{3} \mathrm{Fe}$. In this short communication we present calculations of the ground state properties of $\mathrm{Pt}_{3} \mathrm{Fe}$ for ferromagnetic, antiferromagnetic $\left(\mathrm{AFM}_{1}\right.$ and $\left.\mathrm{AFM}_{2}\right)$ and noncollinear magnetic $(\mathrm{NCM})$ order.

\footnotetext{
* corresponding author; e-mail: jozef.deniszczyk@us.edu.pl
}

\section{Computational details}

The electronic structure calculations were performed using the WIEN2k code of P. Blaha et al. [5] working within Full Potential Linear Augmented Plane Wave (FP-LAPW) method based on the Density Functional Theory (DFT) formalism. The gradient corrected (within Generalized Gradient Approximation) Local Spin Density approximation (LSDA-GGA) for the exchange correlation (XC) potential was used in the form developed in [6]. The enhanced Coulomb correlation interaction for the $3 d$ states of iron atom was included into the $\mathrm{XC}$ potential along the so called LSDA $+\mathrm{U}$ approach [7]. The core electronic states of iron atoms were partitioned into true core states $(1 s, 2 s, 2 p)$, which were treated atomic-like in the self-consistent muffin-tin (MT) spherical potential, and weakly bound semicore states $(3 s, 3 p$ for $\mathrm{Fe}$ ) considered as Local Orbitals (LO) [8] and treated in the full (nonspherical) potential. For the core states, the fully relativistic DFT formalism was employed. For the valence and LO states the scalar-relativistic approximation was used and the spin-orbit (SO) interaction for these states was taken into account within the secondvariational approach [8]. The calculations for the NCM order were performed with the use of the noncollinear version of WIEN2k code [9] with the same computational assumptions (XC potential, SO interaction, electronic states separation, etc.). Within the NCM approach the spin-orbit coupling term was added directly to the scalarrelativistic Hamiltonian.

For all studied phases the MT sphere radii of $1.164 \AA$ for $\mathrm{Fe}$ and $1.27 \AA$ for $\mathrm{Pt}$ were chosen. The set of the $\mathbf{k}$ vectors and the values of $R K_{\max }$ factor were chosen to gain the total energy precision of $10^{-5}$ Ryd per formula unit and the electric field gradient (EFG) relative error less then $1 \%$. All calculations were performed for the experimental lattice parameters. Calculations have shown that for $\mathrm{AFM}_{1}$ and NCM-type of magnetic order some forces acting on atoms occur but their amplitudes are negligible (less than $2 \mathrm{meV} / \mathrm{nm}$ ). In this circumstances 
we neglected the relaxation of atomic positions and applied the standard Wyckoff atomic positions: 3(c) and 1(a) for Pt and Fe atom, respectively.

The EFG tensor was calculated using the formula derived by $\mathrm{M}$. Weinert [10] for the shape model of periodic charge distribution used in the FP-LAPW method. The formula divides the EFG into contribution of electrons located inside MT sphere (local EFG) and the part dependent on the charge distributed outside the MT sphere, called lattice EFG [11]. In our calculations the contribution to EFG of true core states is omitted but, as argued in [11], it is negligible in metals. Some details of the M. Wainert formulation we discussed in [12].

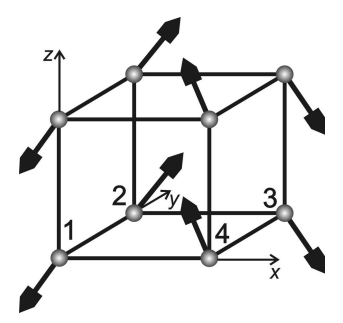

Fig. 1. Noncollinear orientation of the magnetic moments of $\mathrm{Fe}$ atoms in $\mathrm{Pt}_{3} \mathrm{Fe}$ [3].

For the orientation of the local magnetic moments we assumed the construction proposed in [3] on the basis of the neutron scattering measurements. The idea behind this construction is that two types of $\mathrm{AFM}_{1}$ correlations, $(1 / 2,0,0)$ and $(0,1 / 2,0)$, (degenerated, as we proved by $a b$ initio calculations) couple giving rise to the noncollinearity in local magnetic moment orientations within the $x-y$ plane. This intermediate type of magnetic order couple in turn with the $\mathrm{AFM}_{2}$-type correlations resulting in the NCM structure of $\mathrm{Pt}_{3} \mathrm{Fe}$. The resulting noncollinear alignment of Fe magnetic moments is shown in Fig. 1. We considered also another noncolinear orientation of local Fe moments in $\mathrm{Pt}_{3} \mathrm{Fe}$ proposed in [13], but our NCM calculations have shown that this type of spin configuration is less preferable because of higher total energy.

\section{Results and discussion}

The essential results of our calculations are collected in Tables I, II and III. Table I shows the calculated magnitude and the relaxed orientations of the local Fe atoms magnetic moments compared with the experimental ones. Magnetic moment of $\mathrm{Fe}$ atoms in $\mathrm{Pt}_{3} \mathrm{Fe}$ with noncollinear magnetic order is slightly higher (by $\sim 0.1 \mu_{B}$ ) then in FM and both AFM states [4]. An enhancement might be the result of disorder introduced by the noncollinear magnetic structure. Because of the $\mathrm{AFM}_{1}$-type component of the NCM structure, the induced magnetic polarization of $\mathrm{Pt}$ atoms depends on their position, similarly as in the case of $\mathrm{AFM}_{1}$-type of magnetic order [4]. The $\mathrm{Pt}$ atoms, located on the faces where the net $x-y$ plane magnetization exists, acquire nonzero magnetic polarization with magnitude of $0.15 \mu_{B}$, what is two times smaller as compared to that obtained for $\mathrm{AFM}_{1}$-type of magnetic order [4]. The reduction of the Pt magnetic moments can be ascribed to the noncollinear magnetic order which results in the lowering of the overall internal exchange magnetic field and leads to the reduction of the spin density polarization induced on $\mathrm{Pt}$ atoms. The orientation of magnetic moments taken from experiment need not be the stationary one and during the constrained NCM calculations it can be kept frozen by the local constraining magnetic field which is added automatically. In order to find the optimized theoretical directions of Fe magnetic moments we allow for relaxation of their orientation leading to the disappearance of local constraining field and minimizing the total energy. The resulted optimized orientation deviates only slightly from the experimental one (Table I).

TABLE I

Magnitudes and directions of the magnetic moment of $\mathrm{Fe}$ atoms in $\mathrm{Pt}_{3} \mathrm{Fe}$ with noncollinear magnetic order.

\begin{tabular}{l|c|c|c}
\hline \hline \multirow{2}{*}{$\begin{array}{l}\text { Atom } \\
\text { Fe }\end{array}$} & \multicolumn{3}{|c}{ Magnetic moment } \\
\cline { 2 - 4 } & $\begin{array}{c}\text { magnitude } \\
{\left[\mu_{B}\right]}\end{array}$ & \multicolumn{2}{|c}{ Spherical angles $(\theta, \varphi)$} \\
\cline { 2 - 4 } & 3.03 & $\left(122.1^{\circ}, 225.9^{\circ}\right)$ & $\left(122^{\circ}, 226^{\circ}\right)$ \\
\hline 1 & 3.03 & $\left(57.7^{\circ}, 314.1^{\circ}\right)$ & $\left(58^{\circ}, 314^{\circ}\right)$ \\
\hline 2 & 3.03 & $\left(122.1^{\circ}, 45.9^{\circ}\right)$ & $\left(122^{\circ}, 46^{\circ}\right)$ \\
\hline 3 & 3.03 & $\left(57.7^{\circ}, 134.1^{\circ}\right)$ & $\left(58^{\circ}, 134^{\circ}\right)$
\end{tabular}

TABLE II

Calculated relative ground state energies of $\mathrm{Pt}_{3} \mathrm{Fe}$ for paramagnetic (PM), ferromagnetic (FM), antiferromagnetic $\left(\mathrm{AFM}_{1}, \mathrm{AFM}_{2}\right)$ and noncollinear $(\mathrm{NCM})$ magnetic order.

\begin{tabular}{l|l|c|c}
\hline \hline \multirow{2}{*}{$\begin{array}{l}\text { Magnetic } \\
\text { order }\end{array}$} & \multirow{2}{*}{$\begin{array}{l}\text { Calculation } \\
\text { method }\end{array}$} & \multicolumn{2}{|c}{$\Delta E$} \\
\cline { 4 - 4 } & & {$[\mathrm{meV} /$ atom $]$} & {$[\mathrm{K} /$ atom $]$} \\
\hline $\mathrm{PM}$ & \multirow{3}{*}{ LSDA + GGA } & 294.2 & 3414 \\
\cline { 4 - 4 } & & 35.2 & 410 \\
\cline { 4 - 4 } & & 12.3 & 143 \\
\hline $\mathrm{AFM}_{2}$ & & 0 & 20 \\
\hline $\mathrm{AFM}_{1}$ & & &
\end{tabular}

The relative ground state energies for all investigated magnetic structures of $\mathrm{Pt}_{3} \mathrm{Fe}$ are shown in Table II. Our calculations have proved that the ground state magnetic order of $\mathrm{Pt}_{3} \mathrm{Fe}$ is of noncollinear type, what confirms the recent neutron spectroscopy measurements [3]. For the AFM type of magnetic order, the sequence of the calculated ground state energies $\left(\mathrm{AFM}_{2}-\right.$ higher, $\mathrm{AFM}_{1}-$ lower energy) correctly reproduce the order of the magnetic phase transition temperatures $\left(T_{N}>T_{S}\right)$. According to the results presented in Table II, at temperatures $\sim 20 \mathrm{~K}<T<\sim 100 \mathrm{~K}$ both types of AF order can coexist. Below $\sim 20 \mathrm{~K}$ the noncollinear magnetic order in $\mathrm{Pt}_{3} \mathrm{Fe}$ develops. This is in qualitative agreement with the experimental data. 
TABLE III

Magnetic hyperfine field $B_{h f}$ and main component of electric field gradient $\left(\vartheta_{z z}\right)$ with separated partial contributions, calculated within the LSDA-GGA approach. The experimental value of $\vartheta_{z z}$ was estimated for $14.41 \mathrm{keV}{ }^{57} \mathrm{Fe}$ nuclear excitation, assuming the asymmetry parameter equal zero and nuclear quadrupole moment of ${ }^{57} \mathrm{Fe}$ equal $0.17 \mathrm{~b}[14]$.

\begin{tabular}{l|c|c|c|c|c|c|c|c}
\hline \hline & \multirow{2}{*}{ XC potential } & \multirow{2}{*}{$B_{h f}[\mathrm{~T}]$} & \multicolumn{5}{|c}{$\vartheta_{z z}\left[\times 10^{21} \mathrm{~V} \mathrm{\textrm {m } ^ { 2 } ]}\right.$} \\
\cline { 4 - 8 } & & & Lattice & $3 p$-cor. & $3 d$-val. & $4 p$-val. & Total \\
\hline $\mathrm{AFM}_{1}$ & LSDA-GGA & 16.2 & -0.001 & -0.290 & 2.120 & 0.030 & 1.859 \\
\hline $\mathrm{AFM}_{2}$ & LSDA-GGA & 18.7 & 0.003 & 0.130 & -0.620 & -0.020 & -0.507 \\
\hline $\mathrm{NCM}$ & LSDA-GGA & 16.5 & 0.011 & 0.172 & -1.113 & 0.006 & -0.924 \\
\hline $\mathrm{NCM}$ & LSDA-GGA+U $(U=4.5 \mathrm{eV})$ & 17.3 & -0.012 & 0.072 & -0.311 & -0.020 & -0.271 \\
\hline Experimental $\vartheta_{z z}$ & $-0.21 \times 10^{21} \mathrm{~V} \mathrm{~m}^{2}$, at $T=13 \mathrm{~K}$
\end{tabular}

The calculated hyperfine parameters in $\mathrm{Pt}_{3} \mathrm{Fe}$ are collected in Table III and compared with experimental data. The analytic formula of M. Weinert for the main component $\vartheta_{z z}$ of the diagonal EFG allows for the analysis of separate contributions of states located at different regions of energy scale and with different electronic orbital quantum numbers. In agreement with previous calculations for metals reported in [11] we found that the lattice $\vartheta_{z z}$ is vanishingly small. The dominant contribution to $\vartheta_{z z}$ at $\mathrm{Fe}$ nuclei comes from the $3 d$-type valence electrons cancelled partially by weakly bound $3 p$ core electrons due to shielding effect. The contribution of valence $\mathrm{Fe}-4 p$ states is negligible. For both AFM phases the calculated asymmetry parameter is equal zero. For the NCM structure the asymmetry parameter was found equal 0.03 within the LSDA-GGA approach, and $\sim 0.1$ when the enhanced Coulomb correlation for the Fe-3d states is taken into account. In our previous paper [4] we presented the detailed discussion of the origin of the electric field gradient observed in $\mathrm{Pt}_{3} \mathrm{Fe}$ despite its regular crystal structure.

\section{Summary}

The ground state total energy analysis confirms the preference for the noncollinear order in $\mathrm{Pt}_{3} \mathrm{Fe}$ at low temperature. The calculated ground state energies for $\mathrm{AFM}_{1}, \mathrm{AFM}_{2}$ and NCM magnetic order follows the sequence of magnetic phase transitions observed experimentally in $\mathrm{Pt}_{3} \mathrm{Fe}$. Our result for the main component of diagonal EFG tensor reproduce quantitatively the experimental value. The best agreement is obtained when the enhanced Coulomb correlation interaction for the Fe-3d states is taken into account.

\section{Acknowledgements}

The work was supported by a research project NN202172335.

\section{References}

[1] Y. Tsunoda, R. Abe, Phys. Rev. B 55. 11507 (1997).

[2] G.E. Bacon, J. Crangle, Proc. R. Soc. London, Ser. A 272, 387 (1959).

[3] S. Yano, Y. Tsunoda, J. Magn. Magn. Mater. 310, 1841 (2007).

[4] J. Deniszczyk, D. Satuła, J. Waliszewski, K. Rećko, W. Olszewski, G. Parzych, K. Szymański, Acta Phys. Pol. A 115, 197 (2009).

[5] Electronic structure calculations of solids using the WIEN2k package for material science; K. Schwarz, P. Blaha, G.K.H. Madsen, Comput. Phys. Commun. 147, 71 (2002).

[6] J.P. Perdew, K. Burke, M. Ernzerhof, Phys. Rev. Lett. 77, 3865 (1996).

[7] A.I. Lichtenstein, V.I. Anisimov, J. Zaanen, Phys. Rev. B 52, R5467 (1995).

[8] D. Singh, Plane waves, pseudopotentials and the LAPW method, Kluwer Academic, 1994.

[9] R. Laskowski, G.K.H. Madsen, P. Blaha, K. Schwarz, Phys. Rev. B 69, 140408 (2004).

[10] M. Weinert, J. Math. Phys. 22, 2433 (1981).

[11] P. Blaha, K. Schwarz, P.B. Dederichs, Phys. Rev. B 37, 2792 (1988).

[12] T. Michalecki, A. Hanc, J. Deniszczyk, W. Borgiel, Acta Phys. Pol. A 114, 1483 (2008).

[13] Y. Tsunoda, D. Tsuchiya, Y. Higashiyama, J. Phys. Soc. Jpn. 72, 713 (2003).

[14] U.D. Wdowik, K. Ruebenbauer, Phys. Rev. B 76, 155118 (2007). 